\title{
Knockdown of IncRNA NORAD inhibits the proliferation, inflammation and fibrosis of human mesangial cells under high-glucose conditions by regulating the miR-485/NRF1 axis
}

\author{
LINNA WANG $^{1 *}$, XIAOYING YUAN ${ }^{1 *}$, LIFENG LIAN $^{2}$, HUALI GUO $^{3}$, \\ HONGXIA ZHANG $^{1}$ and MINGHUI ZHANG ${ }^{1}$ \\ ${ }^{1}$ Department of Nephrology, ${ }^{2}$ Laboratory Department and ${ }^{3}$ Blood Purification Center, \\ Shengli Oilfield Central Hospital, Dongying, Shandong 257000, P.R. China
}

Received April 10, 2020; Accepted February 11, 2021

DOI: $10.3892 / \mathrm{etm} .2021 .10306$

\begin{abstract}
Long non-coding RNAs (lncRNAs) serve major roles in diabetic nephropathy (DN). The present study investigated the regulatory mechanism of lncRNA non-coding RNA activated by DNA damage (NORAD) on DN in vitro. Reverse transcription-quantitative polymerase chain reaction (RT-qPCR) was used to detect the expression of lncRNA NORAD, microRNA-485 (miR-485) and nuclear respiratory factor 1 (NRF1) in the tissues of patients with DN and high-glucose (HG)-induced human mesangial cells (HMCs). The viability of HMCs was determined using an MTT assay. The levels of inflammatory [tumour necrosis factor (TNF)- $\alpha$, interleukin (IL)-1 $\beta$ and IL-6] and fibrotic [type IV collagen (Col. IV), fibronectin (FN) and plasminogen activator inhibitor 1 (PAI-1)] factors in HMCs were measured by ELISA. The interactions between miR-485 and NORAD/NRF1 were predicted using StarBase and miRDB softwares and confirmed by a dual-luciferase reporter assay. Western blot analysis was utilized to measure NRF1 protein levels. lncRNA NORAD was highly expressed in tissues and HG-induced HMCs. NORAD knockdown suppressed cell viability in HG-induced HMCs. The levels of the inflammatory and fibrotic factors in HG-induced HMCs were inhibited by NORAD knockdown. miR-485 was the direct target of NORAD. NORAD reversed the inhibitory effects of miR-485 on HG-induced HMCs. Furthermore, NRF1 was the target gene of miR-485. Downregulation of miR-485 and upregulation
\end{abstract}

Correspondence to: $\mathrm{Dr}$ Minghui Zhang, Department of Nephrology, Shengli Oilfield Central Hospital, 31 Jinan Road, Dongying, Shandong 257000, P.R. China

E-mail: zhangminghui244@163.com

*Contributed equally

Key words: diabetic nephropathy, non-coding RNA activated by DNA damage, microRNA-485, nuclear respiratory factor 1 , high-glucose of NRF1 reversed the inhibitory effects of NORAD knockdown on HG-induced HMCs. NORAD knockdown inhibited HG-induced HMC proliferation, inflammation and fibrosis by regulating miR-485/NRF1, providing a possible therapeutic strategy for DN.

\section{Introduction}

Diabetic nephropathy (DN) has long been considered the most pervasive and serious diabetic chronic complication (1). Currently, DN is mostly treated with drugs, including rosiglitazone (2), tripterysium glycosides (3) and alprostadil (4). However, these drug treatments have limitations and adverse reactions, including leukopenia, gastrointestinal reactions, irregular menstruation and abnormal liver function $(5,6)$. Therefore, the investigation into novel treatment strategies for DN remains necessary.

Long non-coding RNAs (IncRNAs) are important regulators of cell proliferation, inflammation and fibrosis in DN $(7,8)$. For instance, lncRNA plasmacytoma variant translocation 1 promotes mesangial cell (MC) proliferation under high-glucose conditions in DN $(9,10)$ and lncRNA nuclear enriched abundant transcript 1 (NEAT1) accelerates the occurrence and development of DN (11). Additionally, non-coding RNA activated by DNA damage (NORAD) is a conserved and abundant lncRNA that preserves genomic stability (12). NORAD has been demonstrated to be an onco-lncRNA in various types of human cancer, including prostate (13), ovarian (14), lung (15) and gastric (16) cancers. lncRNA NORAD is also involved in DN progression (17). Qi et al (17) demonstrated that the NORAD/miR-520h/Toll-like receptor 4 regulatory loop promotes the proliferation and inhibits the apoptosis of glomerular MCs, thereby aggravating the progression of DN. However, the possible effects of NORAD on the inflammation and fibrosis of DN and the underlying regulatory mechanisms remain to be fully revealed.

Emerging evidence has indicated that lncRNAs serve roles as competing endogenous RNAs (ceRNAs) for miRNAs and regulate the expression of their target genes in certain diseases (18). miRNAs are involved in cellular processes, including cell viability and apoptosis (19). Furthermore, 
miRNAs act as efficient inhibitors in DN progression (20). For example, miR-544 attenuates diabetic renal injury by suppressing glomerulosclerosis and inflammation (21). miR-320a may be a potential curative target in DN (22). miR-874 overexpression alleviated renal injury in DN rats (23). Notably, miR-485 may serve as a regulator of inflammatory and fibrotic responses (24). In a recent study, miR-485 suppressed MC inflammation and proliferation in an in vitro model of DN (25). Additionally, lncRNAs may act as competing endogenous RNAs or sponges of miRNAs. NORAD has been reported to regulate numerous miRNAs in several types of human disease, including miR-136-5p in retinoblastoma (26), miR-144-3p in hepatocellular carcinoma (27), miR-520a-3p in non-small cell lung cancer (28) and miR-214 in gastric cancer (16). However, the regulatory mechanisms between IncRNA NORAD and miR-485 have not yet been reported.

The present study investigated the effects of lncRNA NORAD on human (H)MC proliferation, inflammation and fibrosis and investigated the regulatory mechanisms between NORAD and miR-485/nuclear respiratory factor 1 (NRF1). The present study revealed that the NORAD/miR-485/NRF1 axis will be a theoretical basis for DN-targeted therapy.

\section{Materials and methods}

Tissue collection. A total of 21 patients with DN without other complications were selected in Shengli Oilfield Central Hospital (Dongying, China) between March 2017 and June 2018. The patients included 11 males and 10 females (age range, 46-64 years; mean age, $54.6 \pm 6.2$ years). These patients had not received treatment within 3 months before admission. Pathological kidney and adjacent normal tissues were obtained by biopsy. Each patient provided written informed consent and agreed to the study being published. The present study was approved by the Ethics Committee of Shengli Oilfield Central Hospital (approval no. Q/ZXYY-ZY-YWB-LL202037).

Cell grouping and transfection. HMCs were purchased from The Cell Bank of Type Culture Collection of the Chinese Academy of Sciences. DMEM containing 10\% FBS (Gibco; Thermo Fisher Scientific, Inc.) was used to culture the cells at $37^{\circ} \mathrm{C}$ in $5 \% \mathrm{CO}_{2}$. Small interfering (siRNA)-negative control (si-NC, 5'-UUCUCCGAACGUGUCACGU-3') and siRNA-NORAD-1/-2 (si-NORAD-1, 5'-AAGCCACCUUUG UGAACAGUA-3'; si-NORAD-2, 5'-GAGAAAUGGUAG AAUGACA-3') were obtained from Sangon Biotech Co., Ltd. NORAD overexpression (ov-NORAD), NRF1 overexpression (ov-NRF1), miR-485 mimics (5'-AGAGGCUGG CCGUGAUGAAUUC-3'), miR-NC (5'-UUCUCCGAACGU GUCACGUTT-3'), miR-485 inhibitor (5'-GUCAUACAC GGCUCUCCUCUCU-3'), and inhibitor NC (5'-CAGUAC UUUUGUAGUACAAA-3') were procured from Guangzhou RiboBio Co., Ltd. HMCs were transfected with the aforementioned agents (all $50 \mathrm{nM}$ ) using a Lipofectamine RNAiMAX kit (Invitrogen; Thermo Fisher Scientific, Inc.) for $48 \mathrm{~h}$ at $37^{\circ} \mathrm{C}$. In addition, the HMCs $\left(6 \times 10^{5}\right.$ cells/well) were further divided into high glucose (HG; $30 \mathrm{mM})$ and normal glucose (NG; $5.5 \mathrm{mM}$ ) groups. At $48 \mathrm{~h}$ after treatment, the cells were harvested to perform the following experiments.
Reverse transcription-quantitative polymerase chain reaction $(R T-q P C R)$. Total RNA was extracted from HMCs using a TRIzol ${ }^{\circledR}$ reagent kit (Invitrogen; Thermo Fisher Scientific, Inc.), according to the manufacturer's protocols. In accordance with the manufacturer's protocols of GoScript ${ }^{\mathrm{TM}}$ reverse transcription system (Promega Corporation), the extracted RNA was initially reverse transcribed into cDNA at $37^{\circ} \mathrm{C}$ for $60 \mathrm{~min}$, followed by $85^{\circ} \mathrm{C}$ for $5 \mathrm{~min}$ and then subjected to qPCR analyses with the Applied Biosystems SYBR $^{\mathrm{TM}}$ Green PCR Master mix (Thermo Fisher Scientific, Inc.). The thermocycling conditions were: Initial denaturation at $95^{\circ} \mathrm{C}$ for $10 \mathrm{~min}$, followed by 40 cycles of $95^{\circ} \mathrm{C}$ for $10 \mathrm{sec}, 60^{\circ} \mathrm{C}$ for $20 \mathrm{sec}$ and $72^{\circ} \mathrm{C}$ for $34 \mathrm{sec}$. U6 or GAPDH was used as the internal reference standard. The primers were designed as follows: NORAD forward: 5'-GGAGAA TCGCTTGAACT-3' and reverse, 5'-CAAACACCCAAT GAATAG-3'; miR-485 forward, 5'-CCAAGCTTCACCCAT TCCTAACAGGAC-3' and reverse, 5'-CGGGATCCGTAG GTCAGTTACATGCATC-3'; NRF1 forward, 5'-TTACTC TGCTGTGGCTGATGG-3' and reverse, 5'-CCTCTGATG CTTGCGTGGTCT-3'; U6 forward, 5'-GCTTCGGCAGCA CATATACTAAAAT-3' and reverse, 5'-CGCTTCACGAAT TTGCGTGTCAT-3' and GAPDH forward, 5'-GAAGGT GAAGGTCGGAGTC-3' and reverse, 5'-GAAGATGGTGAT GGGATTTC-3'. Gene expression was quantified using the $2^{-\triangle \Delta \mathrm{Cq}}$ method (29).

Western blot analysis. Radioimmunoprecipitation assay buffer (Beyotime Institute of Biotechnology) containing protease inhibitors was used to extract proteins from cells. The protein concentrations were determined using a bicinchoninic acid Protein assay kit (Thermo Fisher Scientific, Inc.). A total of $50 \mu \mathrm{g}$ of protein/lane was separated via 10\% SDS-PAGE (Boster Biological Technology). The resolved proteins were then transferred onto polyvinylidene fluoride membranes. Blocking was performed using $5 \%$ bovine serum albumin (Thermo Fisher Scientific, Inc.) at room temperature for 2 h. Following blocking, the membranes were incubated overnight at $4^{\circ} \mathrm{C}$ with primary antibodies against NRF1 (1:1,000; cat. no. ab175932; Abcam) and GAPDH (1:1,000; cat. no. ab8245; Abcam). Next, the membranes were washed three times in TBS-Tween-20 (0.05\%). The secondary antibody horseradish peroxidase-conjugated anti-mice immunoglobulin G (1:2,000; cat. no. ab6728; Abcam) was added and incubated for $1 \mathrm{~h}$ at room temperature. GADPH was used as the internal reference. The membranes were developed using an ECL reagent (Thermo Fisher Scientific, Inc.) under Gel-Pro analyzer (version 4.0; Media Cybernetics, Inc.).

Cell viability assay. MTT assays were used to determine HMC viability. Cells were seeded onto a 96 -well plate with $2 \times 10^{5}$ cells/well and cultured in serum-free medium overnight at $37^{\circ} \mathrm{C}$. Subsequently, cells were incubated under the designated glucose conditions for $24,48,72$ and $96 \mathrm{~h}$ at $37^{\circ} \mathrm{C}$. Next, $20 \mu \mathrm{l}$ MTT (Merck KGaA) was added to each well and the cells were incubated for another $2 \mathrm{~h}$ at $37^{\circ} \mathrm{C}$. The supernatant was removed and the formazan crystals were dissolved using DMSO (150 $\mu \mathrm{l} /$ well). The absorbance at $450 \mathrm{~nm}$ was analysed using a Multiskan Spectrum microplate reader (Thermo Fisher Scientific, Inc.). 

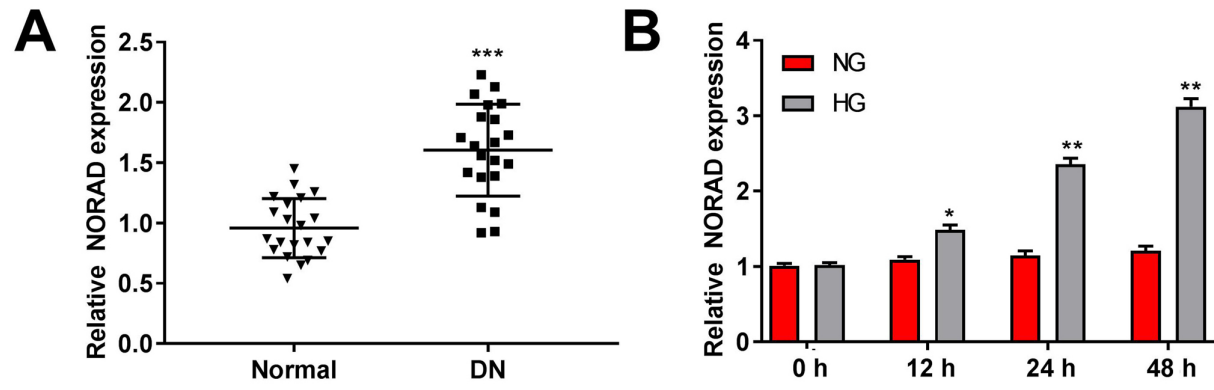

Figure 1. Expression of NORAD in DN tissues and HMCs. (A) Expression of NORAD in DN tissues $(\mathrm{n}=21)$ and adjacent normal tissues ( $\mathrm{n}=21)$ determined by RT-qPCR. ${ }^{* * *} \mathrm{P}<0.001$ vs. normal. (B) Expression of NORAD in HG-stimulated HMCs and NG HMCs determined by RT-qPCR. ${ }^{*} \mathrm{P}<0.05$, ${ }^{* * *} \mathrm{P}<0.01$ vs. NG. NORAD, non-coding RNA activated by DNA damage; DN, diabetic nephropathy; HMCs, human mesangial cells; RT-qPCR, reverse transcription-quantitative polymerase chain reaction; HG, high glucose; NG, normal glucose.

ELISA. According to manufacturer protocols, the levels of inflammatory [tumour necrosis factor (TNF)- $\alpha$ (cat. no. 70-EK182HS-96), interleukin (IL)-1 $\beta$ (cat.no.70-EK101BHS-96)andIL-6(cat.no.70-EK106/2-96)and fibrotic [type IV collagen (Col. IV) (cat. no. RK-009-001-106), fibronectin (FN; cat. no. RK-KOA0169) and plasminogen activator inhibitor 1 (PAI-1) (cat. no. 70-EK1136-96)] factors in HMCs were measured using specific ELISA kits purchased from Multisciences Biotech, Ltd. The absorbance at $450 \mathrm{~nm}$ was determined using a Multiskan Spectrum microplate reader (Thermo Fisher Scientific, Inc.).

Targetprediction. The miRNA targets of NORAD were predicted using StarBase software version 2.0 (http://starbase.sysu.edu. $\mathrm{cn} /$ ), and 272 targets were predicted. Among these miRNA targets, miR-485 was selected for the following assays due to its important role in DN (25) and unknown regulatory association with NORAD. In addition, the mRNA targets of miR-485 were predicted using miRDB software version 3.0 (http://mirdb.org/), and 1,646 targets were predicted. NRF1 was selected for the following assays due to its important role in renal diseases $(30,31)$.

Dual-luciferase reporter assay. To verify the direct interactions between miR-485 and NORAD/NRF1, a dual-luciferase reporter assay was performed. Briefly, the mutant type (MUT) and wild type (WT) of NORAD/NRF1 binding sequences were cloned into the pGL3-promoter (Promega Corporation) to generate the recombinant vectors pGL3-NORAD-WT/-MUT or pGL3-NRF1-WT/-MUT. Cells were co-transfected with the aforementioned recombinant vectors and miR-485 mimic/miR-NC (all $50 \mathrm{nM}$ ) using Lipofectamine ${ }^{\circledR} 3000$ (Invitrogen; Thermo Fisher Scientific, Inc.) at $37^{\circ} \mathrm{C}$ for $48 \mathrm{~h}$. The supernatant was used to measure relative luciferase activity on a Dual-Luciferase Reporter assay system (Promega Corporation). The activity of firefly luciferase was normalized to that of Renilla luciferase.

Statistical analysis. SPSS version 20.0 (IBM Corp.) and GraphPad Prism version 5.01 (GraphPad Software, Inc.) were used to perform the statistical analyses. Data are expressed as the mean \pm standard deviation. Student's t-tests were used to assess the differences between groups (paired, Figs. 1A, 3D and 5C; unpaired, Figs. 1B, 3E and 5D). One-way analysis of variance (ANOVA) was used to investigate the differences among multiple groups. Following ANOVA, pairwise comparisons were performed using Tukey's multiple comparisons tests. $\mathrm{P}<0.05$ was considered to indicate a statistically significant difference. All experiments were conducted in triplicate in at least three independent experiments.

\section{Results}

NORAD is highly expressed in DN tissues and HG-stimulated $H M C s$. The NORAD expression in DN and normal tissues was detected by RT-qPCR. The results demonstrated higher expression in DN tissues compared with in normal tissues $(\mathrm{P}<0.001$; Fig. 1A). Meanwhile, NORAD expression was significantly increased in HG-stimulated HMCs compared with in the NG group ( $\mathrm{P}<0.05$; Fig. 1B).

Knockdown of NORAD inhibits HG-induced HMC proliferation, inflammation and fibrosis. To investigate the possible role of NORAD in DN pathogenesis in vitro, si-NORAD-1/-2 was initially transfected into HMCs to detect the silencing efficiency. As illustrated in Fig. 2A, RT-qPCR demonstrated significantly decreased NORAD expression following transfection of si-NORAD-1 and si-NORAD-2 $(\mathrm{P}<0.01)$. Following treatment with $\mathrm{HG}, \mathrm{NORAD}$ expression was also significantly decreased in the $\mathrm{HG}+$ si-NORAD-1 and $\mathrm{HG}+$ si-NORAD-2 groups $(\mathrm{P}<0.01$; Fig. $2 \mathrm{~B})$. si-NORAD-1 was selected in the following experiments due to its greater silencing efficiency. The results of the MTT assay demonstrated increased $\mathrm{HMC}$ viability in the $\mathrm{HG}+$ si-NC group compared with in the NG group, whilst cell activity was partially inhibited in the $\mathrm{HG}+$ si-NORAD group compared with in the $\mathrm{HG}$ + si-NC group $(\mathrm{P}<0.05$; Fig. $2 \mathrm{C})$. Similarly, the ELISA results demonstrated highly increased levels of inflammatory factors (IL-6, IL-1 $\beta$ and TNF- $\alpha$ ) in the $\mathrm{HG}+$ si-NC group compared with those in the NG group, which was attenuated in the $\mathrm{HG}+$ si-NORAD group $(\mathrm{P}<0.01$; Fig. $2 \mathrm{D}-\mathrm{F})$. The levels of PAI-1, Col. IV and FN were higher in the $\mathrm{HG}+$ si-NC group compared with in the NG group, which was partially reversed in the $\mathrm{HG}+$ si-NORAD group $(\mathrm{P}<0.01$; Fig. $2 \mathrm{G}-\mathrm{I})$. These results indicated that NORAD knockdown suppressed HMC proliferation, inflammation and fibrosis in vitro.

NORAD targets miR-485. Analysis using StarBase software demonstrated potential binding sequences between lncRNA 
A
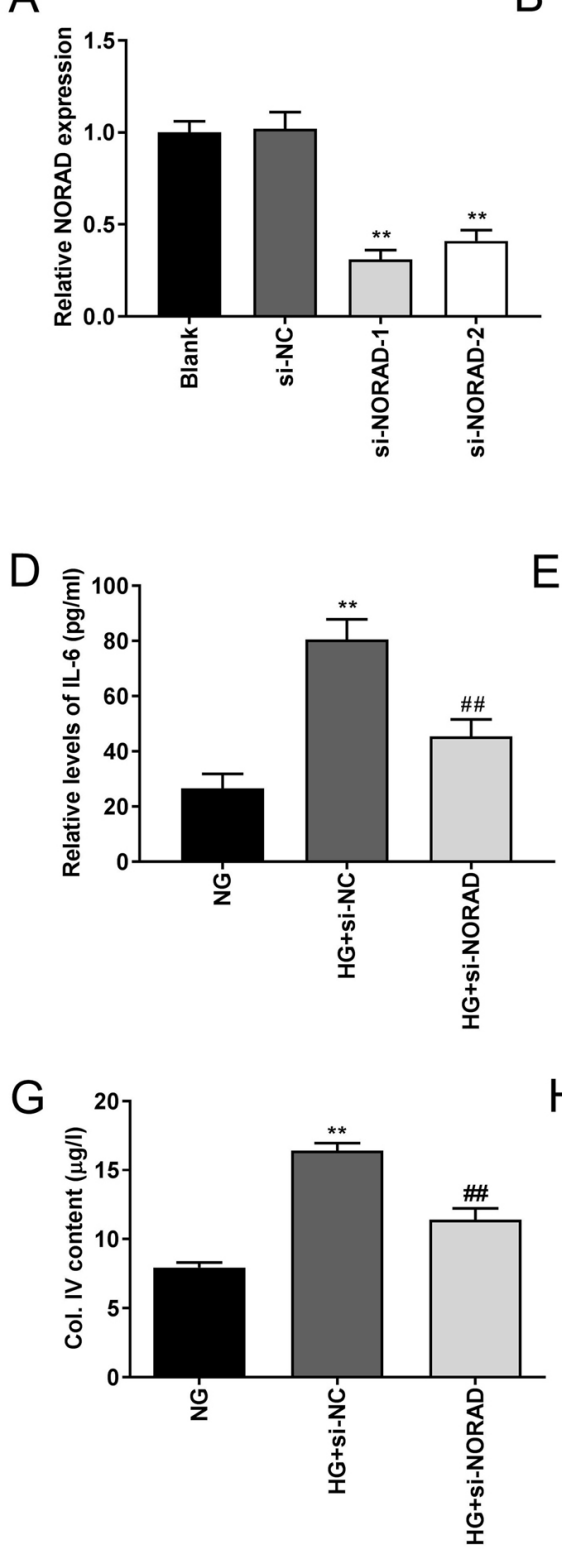

$\mathrm{B}$

E
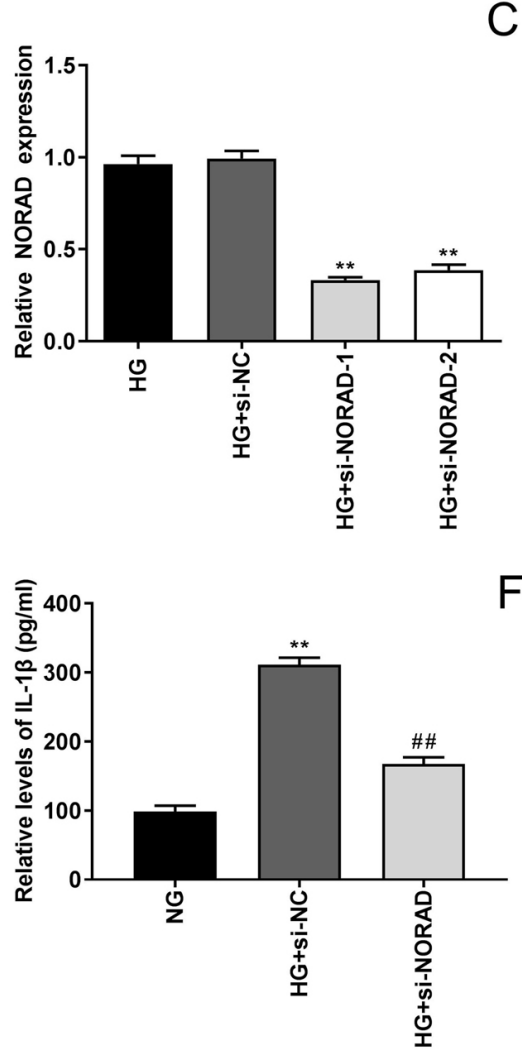

$\mathrm{H}$

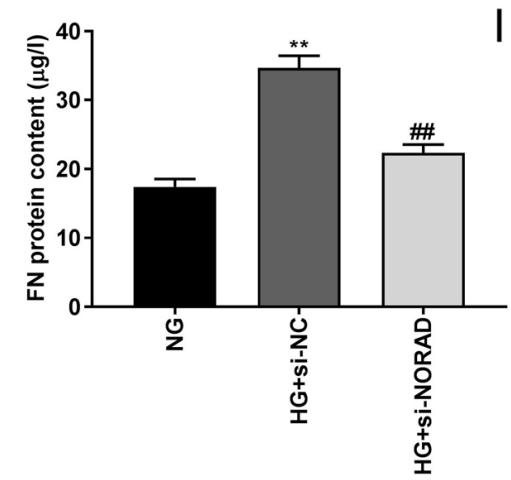

C

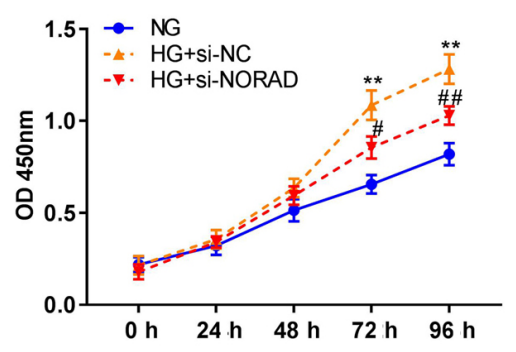

$\mathrm{F}$
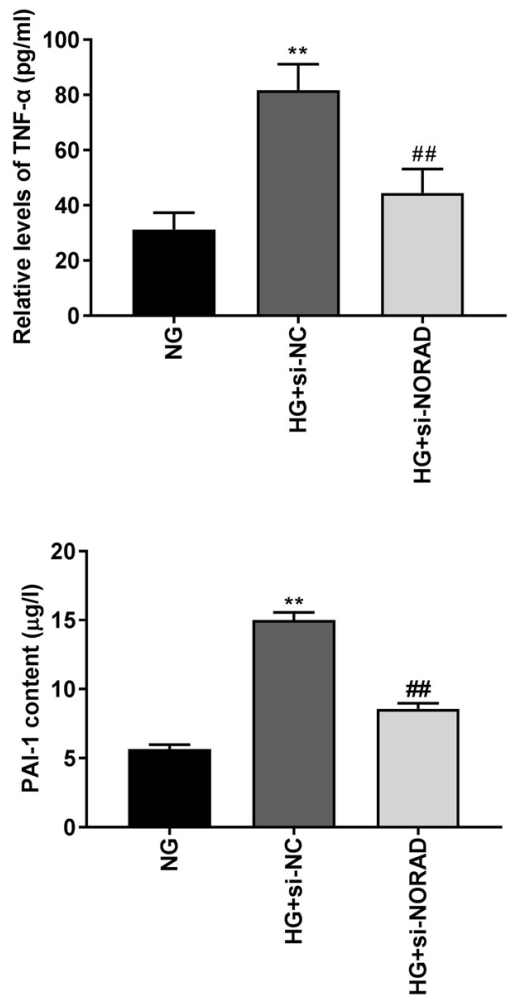

Figure 2. Knockdown of NORAD inhibits proliferation, inflammation and fibrosis in HG-induced HMCs. (A) Expression of NORAD determined by RT-qPCR following transfection of si-NORD-1/-2. ${ }^{* *} \mathrm{P}<0.01$ vs. si-NC. (B) Expression of NORAD detected by RT-qPCR following transfection of si-NORD-1/-2 under HG conditions. ${ }^{* *} \mathrm{P}<0.01$ vs. HG + si-NC. (C) Viability of HMCs measured using MTT assay. ${ }^{* *} \mathrm{P}<0.01$ vs. NG; ${ }^{\#} \mathrm{P}<0.05,{ }^{\# \#} \mathrm{P}<0.01 \mathrm{vs}$. HG + si-NC. Levels of (D) IL-6, (E) IL-1 $\beta$, (F) TNF- $\alpha$, (G) Col. IV, (H) FN and (I) PAI-1 in HMCs measured via ELISA. ${ }^{* *} \mathrm{P}<0.01$ vs. NG; \# P<0.01 vs. HG + si-NC. NORAD, non-coding RNA activated by DNA damage; NG, normal glucose; HG, high glucose; HMCs, human mesangial cells; RT-qPCR, reverse transcription-quantitative polymerase chain reaction; si, small interfering RNA; NC, negative control; OD, optical density; TNF, tumour necrosis factor; IL, interleukin; Col. IV, type IV collagen; FN, fibronectin; PAI-1, plasminogen activator inhibitor 1.

NORAD and miR-485 (Fig. 3A). The luciferase activity in the NORAD WT/miR-485 mimics group was decreased compared with that in the NORAD WT/miR-NC group $(\mathrm{P}<0.01$; Fig. 3B). miR-485 expression was significantly downregulated in the $\mathrm{HG}+$ si-NC group compared with in the NG group, which was attenuated in the $\mathrm{HG}+$ si-NORAD group $(\mathrm{P}<0.01$; Fig. 3C). RT-qPCR assays demonstrated decreased miR-485 expression in DN tissues compared with in normal tissues $(\mathrm{P}<0.001$; Fig. 3D). The expression of miR-485 was also decreased in the HG group compared with in the NG group $(\mathrm{P}<0.01$; Fig. $3 \mathrm{E})$. These data indicated a negative regulatory association between NORAD and miR-485.
NORAD reverses the inhibitory effects of miR-485 on $H G$-induced HMCs. Next, miR-485 mimics, miR-485 inhibitor and miR-485 mimics + ov-NORAD were transfected into HMCs to detect the transfection efficiency. The expression of miR-485 was upregulated in the miR-485 mimics group compared with in the miR-NC group, whilst the inverse was observed in the miR-485 inhibitor group compared with the inhibitor NC group $(\mathrm{P}<0.01$; Fig. 4A). Meanwhile, compared with the miR-485 mimics + ov-NC group, miR-485 expression in the miR-485 mimics + ov-NORAD group was partially inhibited ( $<<0.01$; Fig. $4 \mathrm{~A})$. NORAD expression was then determined following transfection. The results of RT-qPCR analysis demonstrated 


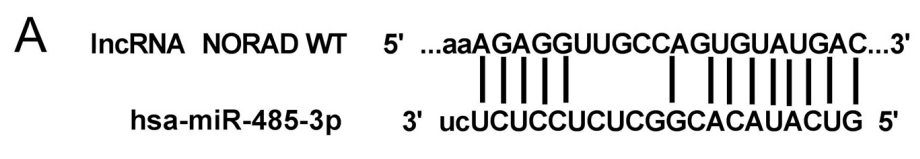

IncRNA NORAD MUT $\quad \mathbf{5}^{\prime}$...aaUCUCCUUGCGACACAUACUC...3'

$\mathrm{B}$

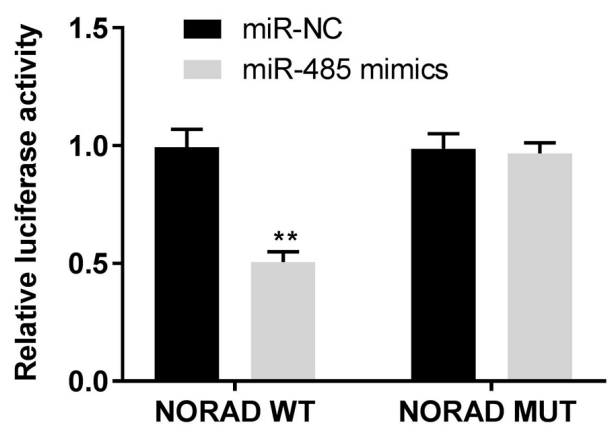

D

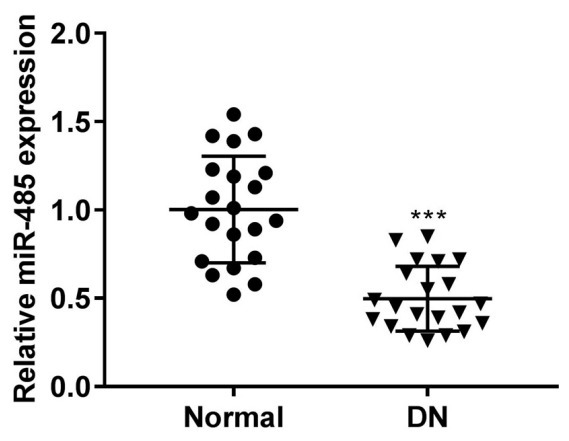

C

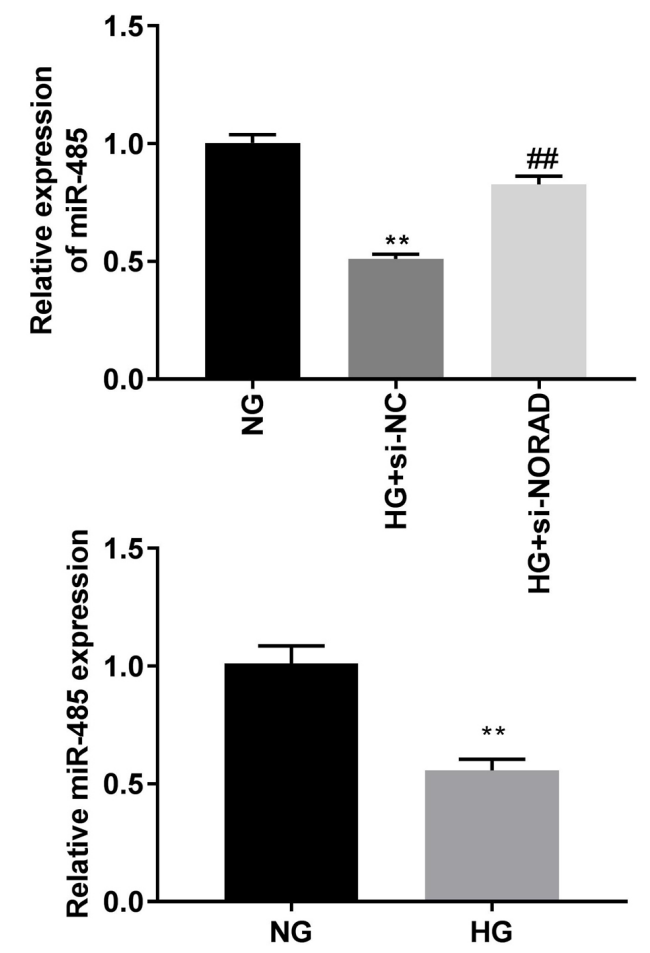

Figure 3. miR-485 is a direct target of NORAD. (A) Predicted complementary binding site of NORAD and miR-485. (B) Luciferase activity in HMCs co-transfected with pGL3-NORAD WT/pGL3-NORAD MUT and miR-485 mimics/NC as determined by a dual luciferase reporter assay. ${ }^{* *} \mathrm{P}<0.01 \mathrm{vs}$. miR-NC. (C) Expression of miR-485 in HMCs detected by RT-qPCR. ${ }^{* *} \mathrm{P}<0.01$ vs. NG; ${ }^{\# /} \mathrm{P}<0.01 \mathrm{vs}$. HG + si-NC. (D) Expression of miR-485 in DN tissues $(\mathrm{n}=21)$ and adjacent normal tissues $(\mathrm{n}=21)$ detected by RT-qPCR. ${ }^{* * *} \mathrm{P}<0.001$ vs. normal. (E) Expression of miR-485 in HG-induced HMCs and NG HMCs detected by RT-qPCR. ${ }^{* *} \mathrm{P}<0.01$ vs. NG. miR, microRNA; NORAD, non-coding RNA activated by DNA damage; HMCs, human mesangial cells; si, small interfering RNA; NC, negative control; RT-qPCR, reverse transcription-quantitative polymerase chain reaction; HG, high glucose; NG, normal glucose; DN, diabetic nephropathy; WT, wild-type; MUT, mutant.

that the expression of NORAD was upregulated by ov-NORAD, but was partially suppressed by ov-NORAD + miR-485 mimics $(\mathrm{P}<0.01$; Fig. 4B). In addition, it was also discovered that miR-485 expression was significantly decreased in the HG group; its expression was upregulated in the $\mathrm{HG}+$ miR-485 mimics group and downregulated in the $\mathrm{HG}+\mathrm{miR}-485$ inhibitor group $(\mathrm{P}<0.01$; Fig. 4C). All the results suggested that miR-485 mimics, miR-485 inhibitor, ov-NORAD and miR-485 mimics + ov-NORAD were successfully transfected into HMCs. The results of the MTT assay demonstrated significantly decreased HG-induced HMC viability in the miR-485 mimics group compared with in the miR-NC group; however, the viability of HG-induced HMCs was partially promoted in the miR-485 mimics + ov-NORAD group compared with in the miR-485 mimics group ( $\mathrm{P}<0.05$; Fig. 4D). Similarly, the ELISA results demonstrated significantly decreased levels of inflammatory (IL-6, IL-1 $\beta$ and TNF- $\alpha$ ) and fibrotic (PAI-1, Col. IV and FN) factors in the miR-485 mimics group compared with in the miR-NC group. Furthermore, overexpression of NORAD attenuated the effects of miR-485 on inflammation and fibrosis in $\mathrm{HG}$-induced HMCs $(\mathrm{P}<0.05$; Fig. 4E and $\mathrm{F})$. Therefore, the results suggested that NORAD may affect the occurrence and development of $\mathrm{DN}$ in vitro by regulating miR-485 expression.

Identification of NRF1 as the target gene of miR-485. Using miRDB software, the potential binding sites between NRF1 and miR-485 were predicted (Fig. 5A). Dual-luciferase reporter assays demonstrated significantly decreased luciferase activity in the NRF1 WT/miR-485 mimics group compared with in the control group. These results indicated that NRF1 was a direct target gene of miR-485 ( $\mathrm{P}<0.01$; Fig. 5B). The RT-qPCR results suggested that the expression of NRF1 was higher in DN tissues compared with in normal tissues $(\mathrm{P}<0.001$; Fig. 5C). Similar to the mRNA levels, the results of the western blot analysis demonstrated downregulation of the NRF1 protein level in the $\mathrm{HG}+\mathrm{miR}-485$ mimics group compared with that in the $\mathrm{HG}+$ miR-NC group $(\mathrm{P}<0.01$; Fig. 5D). These results suggested that miR-485 inhibited NRF1 expression. 

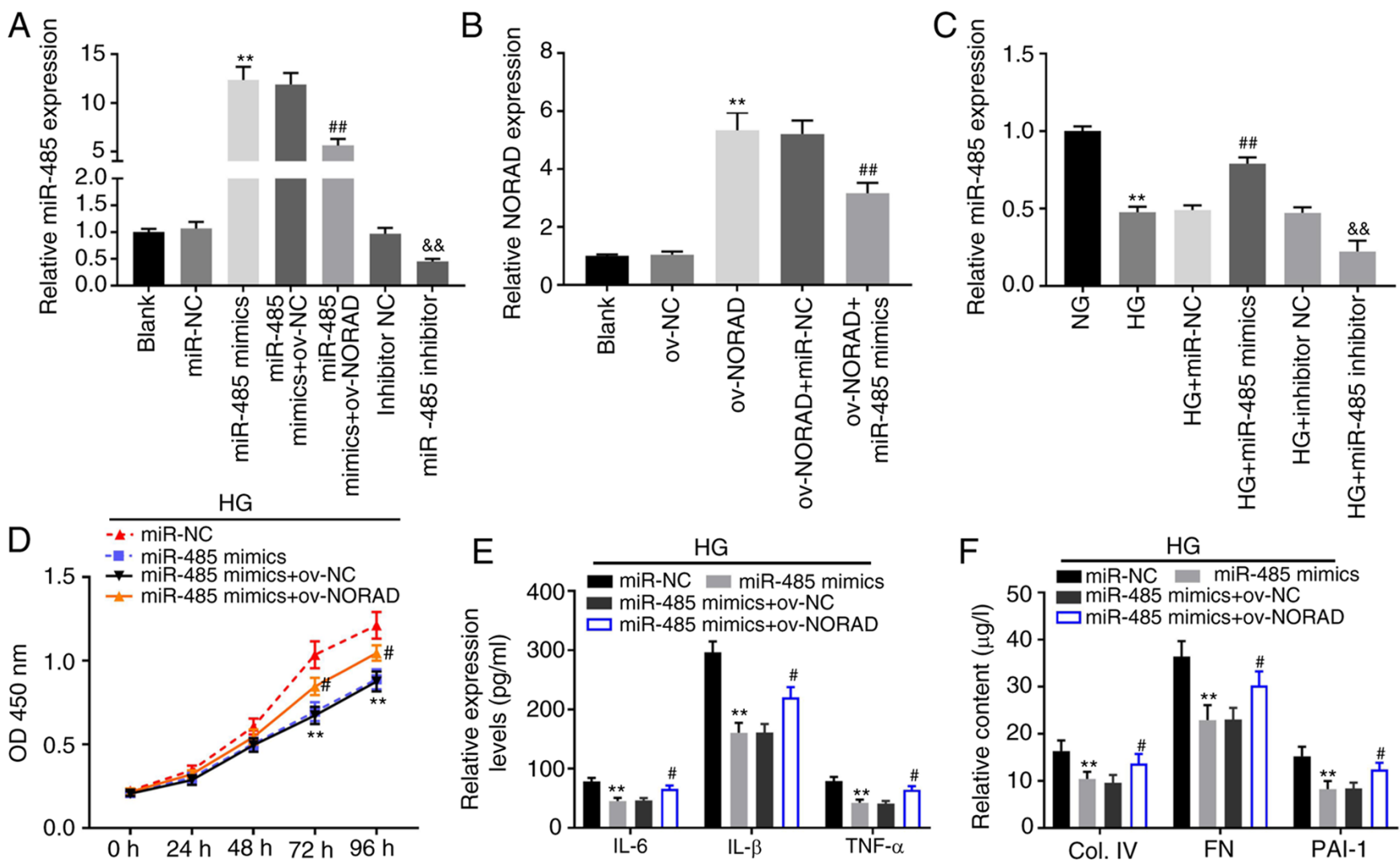

Figure 4. NORAD reverses the inhibitory effects of miR-485 on HG-induced HMCs. (A) Expression of miR-485 following transfection of miR-485 mimics/inhibitor or miR-485 mimics + ov-NORAD detected by RT-qPCR. ${ }^{* *} \mathrm{P}<0.01$ vs. miR-NC; ${ }^{\# \#} \mathrm{P}<0.01$ vs. miR- 485 mimics + ov-NC; ${ }^{\&} \mathrm{P}<0.01$ vs. inhibitor NC. (B) Expression of NORAD following transfection of ov-NORAD or ov-NORAD + miR-485 mimics detected by RT-qPCR. *P $<0.01$ vs. ov-NC; ${ }^{\# \#} \mathrm{P}<0.01$ vs. ov-NORAD + miR-NC. (C) Expression of miR-485 in HG-induced HMCs detected by RT-qPCR. ${ }^{* *} \mathrm{P}<0.01$ vs. NG; ${ }^{\# \#} \mathrm{P}<0.01 \mathrm{vs}$. HG + miR-NC; \&\&P $<0.01$ vs. HG + inhibitor NC. (D) Viability of HG-induced HMCs measured by an MTT assay. ${ }^{* *} \mathrm{P}<0.01$ vs. miR-NC; ${ }^{*} \mathrm{P}<0.05 \mathrm{vs}$. miR-485 mimics. (E) Levels of IL-6, IL-1 $\beta$ and TNF- $\alpha$ in HG-induced HMCs measured via ELISA. *" $\mathrm{P}<0.01$ vs. miR-NC; $\mathrm{P}<0.05$ vs. miR-485 mimics. (F) Contents of Col. IV, FN and PAI-1 in HG-induced HMCs measured via ELISA *** $\mathrm{P}<0.01$ vs. miR-NC; "P<0.05 vs. miR-485 mimics. miR, microRNA; NORAD, non-coding RNA activated by DNA damage; NG, normal glucose; HG, high glucose; HMCs, human mesangial cells; RT-qPCR, reverse transcription-quantitative polymerase chain reaction; ov, overexpression vector; NC, negative control; OD, optical density; TNF, tumour necrosis factor; IL, interleukin; Col. IV, type IV collagen; FN, fibronectin; PAI-1, plasminogen activator inhibitor 1.

A

NRF1 WT $5{ }^{\prime}$...AUAUAUAUAUAUAUAUGUAUGAA...3' hsa-miR-485-3p 3' ucUCUCCUCUCGGCACAUACÚG 5'

NRF1 MUT $5^{\prime}$...AUAUAUAUAUAUAUAACAUACUA...3'

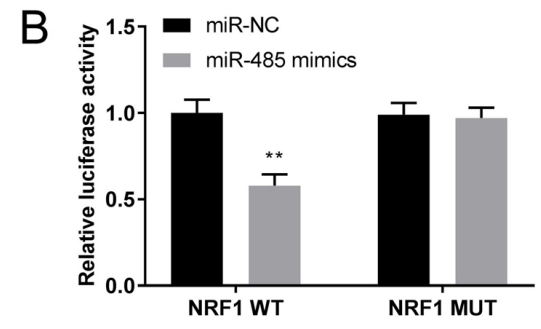

C

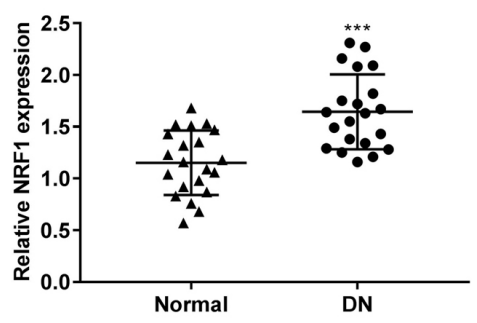

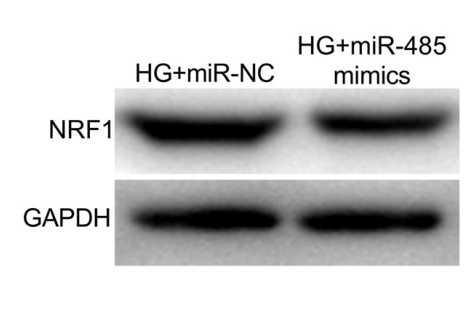

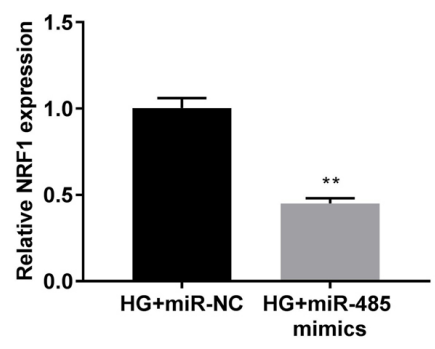

Figure 5. NRF1 is a target gene of miR-485. (A) Predicted complementary binding site of NRF1 and miR-485. (B) Luciferase activity in HMCs co-transfected with pGL3-NRF1 WT/pGL3-NRF1 MUT and miR-485 mimics/NC determined by a dual luciferase reporter assay. ${ }^{* *} \mathrm{P}<0.01$ vs. mimics NC. (C) Expression of NRF1 in DN tissues ( $\mathrm{n}=21)$ and adjacent normal tissues $(\mathrm{n}=21) .{ }^{* * * *} \mathrm{P}<0.001$ vs. normal. (D) Protein expression of NRF1 in HMCs detected via western blot analysis. ${ }^{* *} \mathrm{P}<0.01$ vs. HG + miR-NC. NRF1, nuclear respiratory factor 1; miR, microRNA; HMCs, human mesangial cells; NC, negative control; DN, diabetic nephropathy; WT, wild-type; MUT, mutant; HG, high glucose. 
A

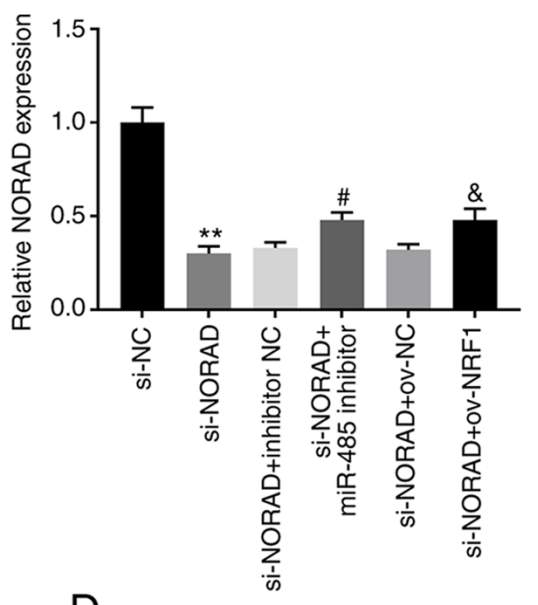

D

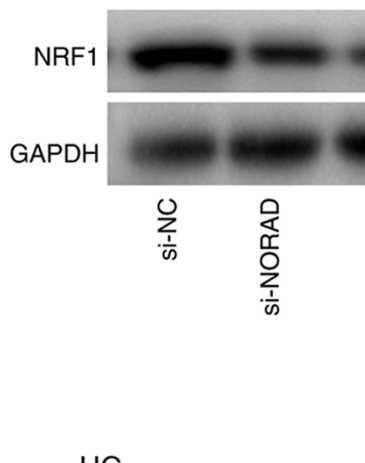

E

- si-NC -m. si-NORAD

$\rightarrow$ si-NORAD+inhibitor NC

$\rightarrow$ si-NORAD+miR-485 inhibitor

1.57 -

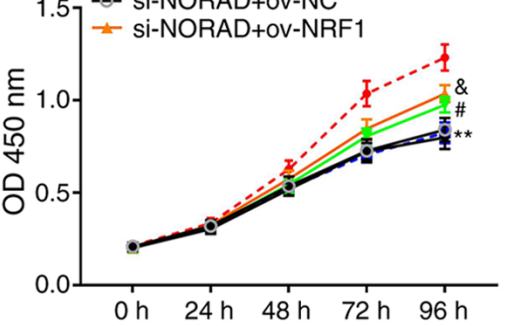

B

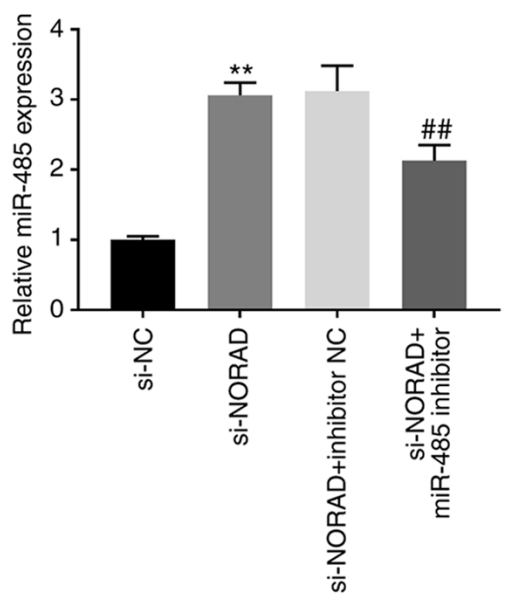

C

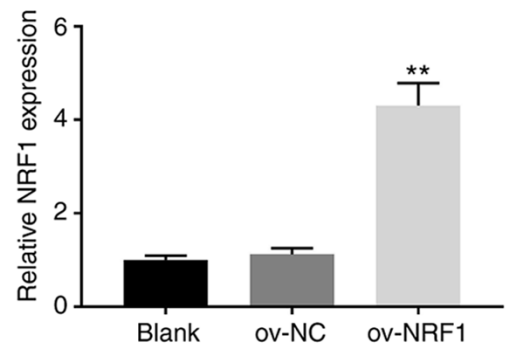

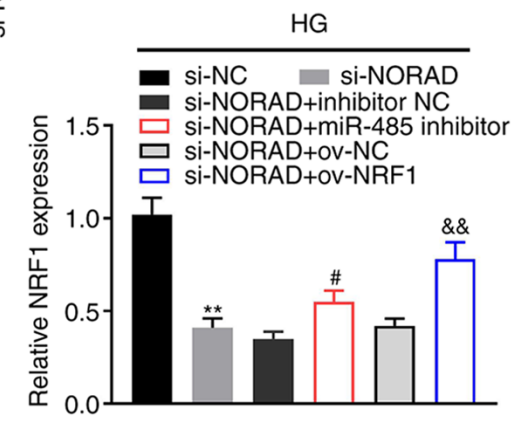

$\mathrm{F}$

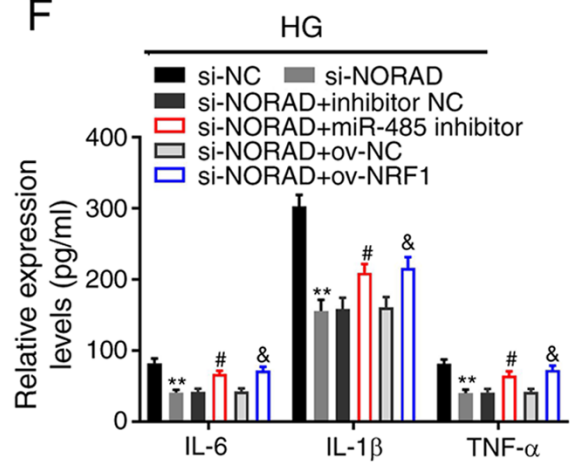

G

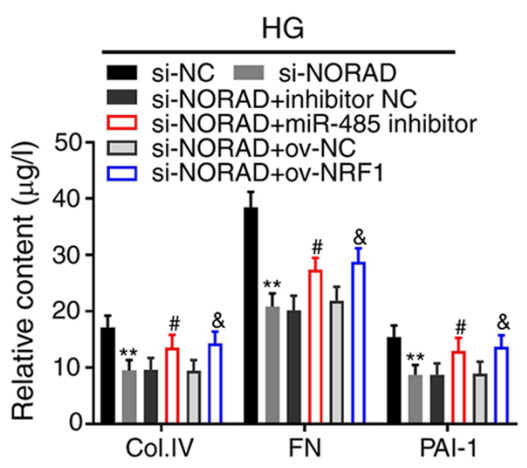

Figure 6. NORAD knockdown inhibits proliferation, inflammation and fibrosis in HG-induced HMCs by regulating the miR-485/NRF1 axis. (A) Expression of NORAD following transfection of si-NORAD, si-NORAD + miR-485 inhibitor or si-NORAD + ov-NRF1 as determined via RT-qPCR. ${ }^{* *} \mathrm{P}<0.01 \mathrm{vs}$. si-NC; ${ }^{\text {"P }}<0.05$ vs. si-NORAD + inhibitor NC; ${ }^{\&} \mathrm{P}<0.05$ vs. si-NORAD + ov-NC. (B) Expression of miR-485 following transfection of si-NORAD or si-NORAD + miR-485 inhibitor as detected via RT-qPCR ${ }^{* *} \mathrm{P}<0.01$ vs. si-NC; ${ }^{\# /} \mathrm{P}<0.01$ vs. si-NORAD + inhibitor NC. (C) Expression of NRF1 following transfection of ov-NRF1 detected by RT-qPCR. ${ }^{* *} \mathrm{P}<0.01$ vs. ov-NC. (D) Protein expression of NRF1 was measured via western blot analysis. ${ }^{* *} \mathrm{P}<0.01$ vs. si-NC; ${ }^{*} \mathrm{P}<0.05$ vs. si-NORAD + inhibitor NC; \&\& $<<0.01$ vs. si-NORAD + ov-NC. (E) Viability of HG-induced HMCs measured by MTT assays. " $\mathrm{P}<0.01$ vs. si-NC; ${ }^{*} \mathrm{P}<0.05$ vs. si-NORAD + inhibitor NC; ${ }^{\circ} \mathrm{P}<0.01$ vs. si-NORAD + ov-NC. (F) Levels of IL-6, IL-1 $\beta$ and TNF- $\alpha$ in HG-induced HMCs as determined via ELISA. ${ }^{* *} \mathrm{P}<0.01$ vs. si-NC; ${ }^{*} \mathrm{P}<0.05$ vs. si-NORAD + inhibitor NC; ${ }^{\circledR} \mathrm{P}<0.05$ vs. si-NORAD + ov-NC. (G) Contents of Col. IV, FN and PAI-1 in HG-induced HMCs as determined via ELISA. ${ }^{* *} \mathrm{P}<0.01$ vs. si-NC; ${ }^{*} \mathrm{P}<0.05$ vs. si-NORAD + inhibitor NC; ${ }^{\circledR} \mathrm{P}<0.05$ vs. si-NORAD + ov-NC. miR, microRNA; NORAD, non-coding RNA activated by DNA damage; NRF1, nuclear respiratory factor 1; HG, high glucose; HMCs, human mesangial cells; RT-qPCR, reverse transcription-quantitative polymerase chain reaction; ov, overexpression vector; si, small interfering RNA; NC, negative control; OD, optical density; TNF, tumour necrosis factor; IL, interleukin; Col. IV, type IV collagen; FN, fibronectin; PAI-1, plasminogen activator inhibitor 1.

NORAD knockdown inhibits proliferation, inflammation and fibrosis in HG-induced HMCs by regulating the miR-485/NRF1 axis. The expression of NORAD and miR-485 following co-transfection was detected. The results of RT-qPCR demonstrated that NORAD expression was significantly increased in the si-NORAD + miR-485 inhibitor and si-NORAD + ov-NRF1 groups compared with their controls (si-NORAD + miR-NC and si-NORAD + ov-NC, respectively; P<0.05; Fig. 6A). Furthermore, the expression of miR-485 was inhibited by co-transfection with si-NORAD + miR-485 inhibitor compared with si-NORAD + inhibitor NC $(\mathrm{P}<0.01$; Fig. 6B). The transfection efficiency of ov-NRF1 was subsequently determined using RT-qPCR, which demonstrated that NRF1 expression was increased by ov-NRF1 ( $<<0.01$; Fig. 6C). The aforementioned results indicated that si-NORAD + miR-485 inhibitor or ov-NRF1 was successfully transfected into HMCs. Western blot analysis demonstrated downregulated NRF1 protein expression following transfection of si-NORAD, while co-transfection with 
miR-485 inhibitor or ov-NRF1 reversed this inhibitory effect $(\mathrm{P}<0.05$; Fig. 6D). MTT assays demonstrated significantly inhibited HG-induced HMC viability in the si-NORAD group compared with that in the si-NC group. Meanwhile, cell viability was partially promoted in the si-NORAD + miR-485 inhibitor and si-NORAD + ov-NRF1 groups compared with in the si-NORAD group $(\mathrm{P}<0.05$; Fig. 6E). Similarly, the ELISA results indicated that NORAD knockdown downregulated the levels of inflammatory (IL-6, IL-1 $\beta$ and TNF- $\alpha$ ) and fibrotic (PAI-1, Col. IV and FN) factors. However, downregulation of miR-485 and upregulation of NRF1 reversed the effects of NORAD knockdown on inflammation and fibrosis in HG-induced HMCs $(\mathrm{P}<0.05$; Fig. $6 \mathrm{~F}$ and $\mathrm{G})$. These results indicated that knockdown of NORAD may suppress HG-induced HMC proliferation, inflammation and fibrosis by regulating miR-485/NRF1.

\section{Discussion}

Increasing evidence has indicated that hyperglycaemia serves a major role in DN (32). Inflammatory and fibrotic reactions in diabetic patients are mainly caused by hyperglycaemia and ultimately accelerate the development of DN $(33,34)$. Recent studies have demonstrated that lncRNAs serve a critical role in DN (25-27). A previous study reported upregulation of lncRNA antisense non-coding mitochondrial RNA-2 in DN tissues and HG-treated MCs (35). Another study observed significantly upregulated expression of lncRNA distal-less homeobox 6, opposite strand 1 (Dlx6os1) in MCs under HG conditions compared with NG conditions (36). Similarly, the present study identified that lncRNA NORAD was highly expressed in HG-stimulated HMCs and DN tissues. Therefore, NORAD may be a pathogenic factor and may serve as a biomarker for the prognosis of DN.

In the last decade, IncRNAs have been identified as important regulators of cell proliferation, inflammation and fibrosis in DN $(13,27,28)$. Hyperglycaemia is widely proposed to affect different types of nephrocytes (32). Furthermore, MC proliferation, inflammation and fibrosis are the three major features of DN $(13,27,28)$. Ma et al (37) reported that downregulation of 1ncRNA NEAT1 inhibited the proliferation, fibrosis and inflammation of mouse MCs in DN. Feng et al (7) observed that lncRNA brown fat lncRNA 1 interference attenuated renal inflammation fibrosis in DN. Furthermore, another study demonstrated that inhibition of IncRNA Dlx6os1 decreased cell proliferation and fibrosis in DN (36). In the present study, NORAD knockdown suppressed HG-stimulated HMC proliferation, inflammation and fibrosis. Similar to the results of the present study, a recent study also demonstrated that knockdown of NORAD decreased cell viability in mouse glomerular mesangial cells in DN (17). However, the previous study only investigated the mechanism of NORAD on cell proliferation. The results of the present study further revealed the involvement of lncRNA NORAD in regulating HMC proliferation, inflammation and fibrosis in DN.

Recent studies have reported that miRNAs act as regulatory factors in various cellular processes. For example, miRNAs affect cell proliferation, apoptosis, stress resistance and angiogenesis (38). Yao et al (23) reported that miR-874 alleviates renal injury and inflammatory response in DN.
Jiang et al (20) observed that miR-342-3p-overexpression suppressed renal interstitial fibrosis in DN. The present study revealed decreased miR-485 expression in HG-stimulated HMCs and DN tissues. Furthermore, miR-485 was the direct target of lncRNA NORAD and inhibited HG-stimulated HMC proliferation, inflammation and fibrosis. Similar to the results of the present study, Wu et al (25) also demonstrated that miR-485 overexpression suppressed HG-induced HMC proliferation. The results demonstrated that miR-485 may inhibit DN progression. The present study also demonstrated that NORAD overexpression attenuated the inhibitory effects of miR-485 on HG-induced HMC proliferation, inflammation and fibrosis. These results suggested that NORAD may affect DN progression by regulating miR-485 expression.

Emerging evidence has suggested that NRF1 is an important regulatory factor in apoptosis (39). Zhang et al (39) reported that NRF1 overexpression inhibited the apoptosis of palmitate-stimulated human cardiac myocytes. Zhang et al (40) also indicated that NRF1 acts as a key regulator of chondrocyte apoptosis in osteoarthritis. The present study demonstrated significantly increased NRF1 expression in DN tissues and showed that transfection of miR-485 mimics into HG-stimulated HMCs inhibited NRF1 expression. Therefore, NRF1 was determined to be the target gene of miR-485. Furthermore, it was demonstrated that NRF1 expression was negatively regulated by $\mathrm{miR}-485$, and that downregulation of miR-485 and upregulation of NRF1 reversed the effects of NORAD-knockdown on HG-induced HMC proliferation, inflammation and fibrosis. The results indicated that NORAD knockdown inhibited $\mathrm{HG}$-induced HMC proliferation, inflammation and fibrosis by regulating miR-485 and NRF1 expression.

In conclusion, the results of the present study revealed that NORAD knockdown inhibited the proliferation, inflammation and fibrosis of HG-induced HMCs by regulating the in vitro expression of miR-485 and NRF1. However, the difference between in vitro and in vivo conditions is a limitation of the present study. Further studies are warranted to elucidate these issues. Despite this limitation, these findings suggest the potential of a novel strategy for treating DN.

\section{Acknowledgements}

Not applicable.

\section{Funding}

No funding was received.

\section{Availability of data and materials}

The datasets used and/or analyzed during the current study are available from the corresponding author on reasonable request.

\section{Authors' contributions}

LW and HG were responsible for the conception and design of the study, and project supervision and management. XY and $\mathrm{HZ}$ performed data acquisition and analysis. LL and MZ were involved in data analysis and visualization. All 
authors confirmed the authenticity of all the raw data, gave final approval of the version to be published, and agreed to be accountable for all aspects of the work. All authors read and approved the final version of the manuscript.

\section{Ethics approval and consent to participate}

Written informed consent was obtained from each patient. The present study was approved by the Ethics Committee of Shengli Oilfield Central Hospital (approval no. Q/ZXYY-ZY-YWB-LL202037).

\section{Patient consent for publication}

Not applicable.

\section{Competing interests}

The authors declare that they have no competing interests.

\section{References}

1. Fox CS, Matsushita K, Woodward M, Bilo HJ, Chalmers J, Heerspink HJ, Lee BJ, Perkins RM, Rossing P, Sairenchi T, et al: Associations of kidney disease measures with mortality and end-stage renal disease in individuals with and without diabetes: A meta-analysis. Lancet 380: 1662-1673, 2012.

2. Zhang L, Zhou Y, Zhou F, Yu X, Liu J, Liu Y, Zhu Y, Wang $\mathrm{W}$ and Chen N: Altered expression of long noncoding and messenger RNAs in diabetic nephropathy following treatment with rosiglitazone. Biomed Res Int 2020: 1360843, 2020.

3. Wang ZS, Qiu T, Liu XH, Zhou JQ, Chen ZB, Wang L, Zhang L, Shen $Y$ and Zhang L: Tripterysium glycosides preconditioning attenuates renal ischemia/reperfusion injury in a rat model. Int Urol Nephrol 48: 213-221, 2016.

4. Xu X, Pan X and Li S: Prospective analysis of the efficacy of beraprost sodium combined with alprostadil on diabetic nephropathy and influence on rennin-angiotensin system and TNF- $\alpha$. Exp Ther Med 19: 639-645, 2020.

5. Yang XM, Yang KN, Gao W and Cao XJ: Analysis of effect and adverse reactions of enalapril on diabetic nephropathy. J Clin Med Pract: 3, 2012

6. Cao Y, Yun N and Zou A: Meta-analysis of ADR induced by tripterysium glycosides tablet. China Pharmacy 29: 125-130, 2018.

7. Feng X, Zhao J, Ding J, Shen X, Zhou J and Xu Z: lncRNA Blnc1 expression and its effect on renal fibrosis in diabetic nephropathy Am J Transl Res 11: 5664-5672, 2019.

8. Gao J, Wang W, Wang F and Guo C: lncRNA-NR 033515 promotes proliferation, fibrogenesis and epithelialto-mesenchymal transition by targeting miR-743b-5p in diabetic nephropathy. Biomed Pharmacother 106: 543-552, 2018.

9. Alvarez ML and DiStefano JK: Functional characterization of the plasmacytoma variant translocation 1 gene (PVT1) in diabetic nephropathy. PLoS One 6: e18671, 2011.

10. Alvarez ML, Khosroheidari M, Eddy E and Kiefer J: Role of microRNA 1207-5P and its host gene, the long non-coding RNA Pvt1, as mediators of extracellular matrix accumulation in the kidney: Implications for diabetic nephropathy. PLoS One 8: e77468, 2013.

11. Li N, Jia T and Li Y: lncRNA NEAT1 accelerates the occurrence and development of diabetic nephropathy by sponging miR-23c. Eur Rev Med Pharmacol Sci 24: 1325-1337, 2020 .

12. Lee S, Kopp F, Chang TC, Sataluri A, Chen B, Sivakumar S, $\mathrm{Yu} \mathrm{H}, \mathrm{Xie} \mathrm{Y}$ and Mendell JT: Noncoding RNA NORAD regulates genomic stability by sequestering PUMILIO proteins. Cell 164: 69-80, 2016.

13. Zhang $\mathrm{H}$ and Guo $\mathrm{H}$ : Long non-coding RNA NORAD induces cell proliferation and migration in prostate cancer. J Int Med Res 47: 3898-3904, 2019.
14. Tong L, Ao Y, Zhang H, Wang K, Wang Y and Ma Q: Long noncoding RNA NORAD is upregulated in epithelial ovarian cancer and its downregulation suppressed cancer cell functions by competing with miR-155-5p. Cancer Med 8: 4782-4791, 2019.

15. Li J, Xu X, Wei C, Liu L and Wang T: Long noncoding RNA NORAD regulates lung cancer cell proliferation, apoptosis, migration, and invasion by the miR-30a-5p/ADAM19 axis. Int J Clin Exp Pathol 13: 1-13, 2020.

16. Tao W, Li Y, Zhu M, Li C and Li P: IncRNA NORAD promotes proliferation and inhibits apoptosis of gastric cancer by regulating $\mathrm{miR}-214 / \mathrm{Akt} / \mathrm{mTOR}$ axis. Onco Targets Ther 12: 8841-8851,2019.

17. Qi H, Yao L and Liu Q: NORAD affects the progression of diabetic nephropathy through targeting miR-520h to upregulate TLR4. Biochem Biophys Res Commun 521: 190-195, 2020.

18. Zhang G, Sun H, Zhang Y, Zhao H, Fan W, Li J, Lv Y, Song Q, Li J, Zhang M and Shi H: Characterization of dysregulated lncRNA-mRNA network based on ceRNA hypothesis to reveal the occurrence and recurrence of myocardial infarction. Cell Death Discov 4: 35, 2018.

19. Chen L, Nan A, Zhang N, Jia Y, Li X, Ling Y, Dai J, Zhang S, Yang Q, Yi Y and Jiang Y: Circular RNA 100146 functions as an oncogene through direct binding to miR-361-3p and miR-615-5p in non-small cell lung cancer. Mol Cancer 18: 13, 2019.

20. Jiang ZH, Tang YZ, Song HN, Yang M, Li B and Ni CL: miRNA-342 suppresses renal interstitial fibrosis in diabetic nephropathy by targeting SOX6. Int J Mol Med 45: 45-52. 2020.

21. Sun T, Liu Y, Liu L and Ma F: MicroRNA-544 attenuates diabetic renal injury via suppressing glomerulosclerosis and inflammation by targeting FASN. Gene 723: 143986, 2020.

22. He M, Wang J, Yin Z, Zhao Y, Hou H, Fan J, Li H, Wen Z, Tang J, Wang Y, et al: MiR-320a induces diabetic nephropathy via inhibiting MafB. Aging (Albany NY) 11: 3055-3079, 2019.

23. Yao T, Zha D, Gao P, Shui $\mathrm{H}$ and $\mathrm{Wu} X$ : MiR-874 alleviates renal injury and inflammatory response in diabetic nephropathy through targeting toll-like receptor-4. J Cell Physiol 234: 871-879, 2018.

24. Chen HO, Zhang L, Tang ZY and Gong ZM: MiR-485-5p promotes the development of osteoarthritis by inhibiting cartilage differentiation in BMSCs. Eur Rev Med Pharmacol Sci 22: 3294-3302, 2018.

25. Wu J, Lu K, Zhu M, Xie X, Ding Y, Shao X, Chen Y, Liu J, Xu M, $\mathrm{Xu}$ Y, et al: miR-485 suppresses inflammation and proliferation of mesangial cells in an in vitro model of diabetic nephropathy by targeting NOX5. Biochem Biophys Res Commun 521: 984-990, 2020.

26. Yang XL, Hao YJ, Wang B, Gu XL, Wang XX and Sun JF: Long noncoding RNA NORAD promotes the progression of retinoblastoma by sponging miR 136-5p/PBX3 axis. Eur Rev Med Pharmacol Sci 24: 4055, 2020.

27. Tian Q, Yan X, Yang L, Liu Z, Yuan Z, Shen Z and Zhang Y: lncRNA NORAD promotes hepatocellular carcinoma progression via regulating miR-144-3p/SEPT2. Am J Transl Res 12: 2257-2266, 2020

28. Wan Y, Yao Z, Chen W and Li D: The lncRNA NORAD/miR-520a-3p facilitates malignancy in non-small cell lung cancer via PI3k/Akt/mTOR signaling pathway. Onco Targets Ther 13: 1533-1544, 2020.

29. Livak KJ and Schmittgen TD: Analysis of relative gene expression data using real-time quantitative PCR and the 2(-Delta Delta C(T)) method. Methods 25: 402-408, 2001.

30. Huang HQ, Ni HF, Ma KL and Zou JH: ANGPTL2 regulates autophagy through the MEK/ERK/Nrf-1 pathway and affects the progression of renal fibrosis in diabetic nephropathy. Am J Transl Res 11: 5472-5486, 2019.

31. Hsieh PF, Liu SF, Hung TJ, Hung CY, Liu GZ, Chuang LY, Chen MF, Wang JL, Shi MD, Hsu CH, et al: Elucidation of the therapeutic role of mitochondrial biogenesis transducers NRF-1 in the regulation of renal fibrosis. Exp Cell Res 349: 23-31, 2016.

32. Kaur H, Chien A and Jialal I: Hyperglycemia induces Toll like receptor 4 expression and activity in mouse mesangial cells: Relevance to diabetic nephropathy. Am J Physiol Renal Physiol 303: F1145-F1150, 2012.

33. Sortica DA, Crispim D, Zaffari GP, Friedman R and Canani LH: The role of ecto-nucleotide pyrophosphatase/phosphodiesterase 1 in diabetic nephropathy. Arq Bras Endocrinol Metabol 55: 677-685, 2011.

34. Burcelin R, Serino M, Chabo C, Blasco-Baque V and Amar J: Gut microbiota and diabetes: From pathogenesis to therapeutic perspective. Acta Diabetol 48: 257-273, 2011 
35. Gao Y, Chen ZY, Wang Y, Liu Y, Ma JX and Li YK: Long non-coding RNA ASncmtRNA-2 is upregulated in diabetic kidneys and high glucose-treated mesangial cells. Exp Ther Med 13: 581-587, 2017.

36. Cheng J, Cheng L, Tang Y, Li H, Peng W and Huang S: Inhibition of lncRNA Dlx6os1 decreases cell proliferation and fibrosis and increases cell apoptosis in diabetic nephropathy. Int J Clin Exp Pathol 11: 3302-3309, 2018

37. Ma J, Zhao N, Du L and Wang Y: Downregulation of lncRNA NEAT1 inhibits mouse mesangial cell proliferation, fibrosis, and inflammation but promotes apoptosis in diabetic nephropathy. Int J Clin Exp Pathol 12: 1174-1183, 2019.
38. Liang YZ, Dong J, Zhang J, Wang S, He Y and Yan YX: Identification of neuroendocrine stress response-related circulating microRNAs as biomarkers for type 2 diabetes mellitus and insulin resistance. Front Endocrinol (Lausanne) 9: 132, 2018.

39. Zhang J, Gu JY, Chen ZS, Xing KC and Sun B: Astragalus polysaccharide suppresses palmitate-induced apoptosis in human cardiac myocytes: The role of Nrf1 and antioxidant response. Int J Clin Exp Pathol 8: 2515-2524, 2015.

40. Zhang M, Wang Z, Li B, Sun F, Chen A and Gong M: Identification of microRNA-363-3p as an essential regulator of chondrocyte apoptosis in osteoarthritis by targeting NRF1 through the p53-signaling pathway. Mol Med Rep 21: 1077-1088, 2020. 